\title{
Nitrogen removal in a cascade of four reactors employing the activated sludge process
}

\author{
M.I. Nelson ${ }^{\mathrm{a}}$ and F.I. Hai ${ }^{\mathrm{b}}$ \\ ${ }^{a}$ School of Mathematics and Applied Statistics, University of Wollongong, Wollongong, NSW 2522, Australia \\ ${ }^{\mathrm{b}}$ School of Civil Mining and Environmental Engineering, University of Wollongong, Wollongong, NSW 2522, \\ Australia \\ Email: $\underline{\text { mnelson@uow.edu.au }}$
}

\begin{abstract}
The activated sludge process is the most widely used process for the biological treatment of domestic and industrial wastewaters. Wastewater treatment plants based on the activated sludge process are in widespread use in developed and developing countries. The activated sludge model number 1 (ASM \#1) is an internationally accepted standard for activated sludge modeling. It describes nitrogen and chemical oxygen demand within suspended-growth treatment processes, including mechanisms for nitrification and denitrification.

We analyse the biological treatment of a wastewater when a cascade of four reactors is used. We assume that each reactor in the cascade has the same volume. Operating conditions are investigated in which the first reactor is not aerated whilst the last two reactors are aerated. The second reactor may either be aerated or not aerated. The process configuration includes one settling unit and one recycle unit. The settling unit is placed after the final reactor of the cascade. Part of its exit stream is wasted and the remainder is fed into the first reactor. The recycle unit is also placed after the final reactor of the cascade. The entirety of its exit stream is fed into the first reactor.
\end{abstract}

The performance of a wastewater treatment plant can be characterised by a number of process parameters. Here we consider the nitrogen concentration in the effluent stream leaving the treatment plant $\left(\mathrm{TN}_{e}\right)$. When the reactor configuration includes a settling unit this is defined by

$$
\mathrm{TN}_{e}=S_{\mathrm{NO}}+S_{\mathrm{NH}}+S_{\mathrm{ND}}
$$

where the state variables on the right hand are the concentration of soluble nitrate and nitrite nitrogen $\left(S_{\mathrm{NO}}\right)$, soluble ammonium nitrogen $\left(S_{\mathrm{NH}}\right)$, and soluble biodegradable organic $\left(S_{\mathrm{ND}}\right)$ respectively.

A combination of direct numerical integration and continuation methods are used to investigate the steadystate behaviour of the system. The governing equations were solved using both matlab (ode15s) and maple (lsode[backfull]). For continuation XPPAUT was used. We take the hydraulic retention time (HRT) as the bifurcation parameter, primarily allowing it to vary over the range $0<\mathrm{HRT}$ (days) $\leq 1$. We investigate how the nitrogen concentration in the effluent stream depends upon the operation of the recycle units and the state of aeration in the second reactor.

Our results are summarised as follows.

1. When the second reactor is aerated the value of the recycle ratio that minimise the nitrogen concentration depends upon the value of the hydraulic retention time. There is a significant range of values for the hydraulic retention time over which the optimal performance is achieved by employing a 'moderate' recycle ratio.

2. When the second reactor is not aerated increasing the value of the recycle ratio always improves performance. Thus the recycle unit should be operated at the maximum attainable value of the recycle ratio.

3. If the maximum attainable value of the recycle ratio is 'low' then the second reactor should be aerated. If this value is 'high' then the second reactor should not be aerated.

Keywords: Activated sludge, dentrification, modelling, nitrification, process modelling 


\section{BACKGROUND TO THE MODEL}

In this section we outline our implementation of the the ASM\#1 in a four-reactor cascade containing both a settling unit and a recycle unit. The settling and recycle units are placed after the final recycle in the cascade and recycle their exit streams into the first reactor of the cascade.

To model the settling unit we use the the ideal settling unit model, also known as the perfect or point settling unit model. In this model the operation of a settling unit is characterised by three parameters. These are its recycle ratio $(R)$, its concentration factor $(\mathcal{C})$, and the fraction of the non-effluent stream leaving the settling unit that is wasted $(w)$. (For a discussion of other approaches to modelling settling units we refer to Nelson et al. (2018)). The recycle unit is characterised by one parameter: a recycle ratio $\left(R_{41}\right)$.

Brevity does not permit a full discussion of the model, including providing the full system of differential equations, a nomenclature, and a list of parameter values. We refer to Henze et al. (1987) for a detailed description of the assumptions underlying the ASM \#1. Our work builds up an earlier investigation into the steady-state behaviour of a single reactor connected to a settling unit. Nelson et al. (2019) list all the differential equations used in that model and provide a full nomenclature and a complete listing of parameter values. Taken together the references (Henze et al., 1987; Nelson et al., 2019) provide all necessary details for the interested reader to flesh out the account of the model provided here. For further details we refer to the book by Makinia (2010) which provides a comprehensive guide to modelling and simulations studies in activated sludge systems. The modelling concepts used in ASM \#1 (and related models) have been reviewed by Hauduc et al. (2013).

\subsection{State variables}

The ASM \#1 model contains differential equations for both soluble and particulate components. There are six soluble components and six particulate components. The soluble components are: inert soluble organic material, readily biodegradable soluble substrate, soluble oxygen, soluble nitrate \& nitrite nitrogen (one component), soluble ammonium $\left(\mathrm{NH}_{4}^{+}\right.$and $\mathrm{NH}$ ) nitrogen, and soluble biodegradable organic nitrogen. The particulate components are: particulate inert organic matter, lowly biodegradable particulate substrate, active heterotrophic particulate biomass, active autotrophic particulate biomass, non-biodegradable particulate products arising from biomass decay, and particulate biodegradable organic nitrogen.

In writing the equations we use the symbol $S_{O, k}$ to denote the concentration of soluble oxygen in the $k$ th reactor $(k=1 \ldots 4)$ and the symbol $S_{j, k}(j=1 \ldots 5)$ to denote the concentration of the five remaining soluble components in the $k$ th reactor. We use the symbol $X_{l, k}(l=1 \ldots 6)$ to denote the concentration of the six particulate components in the $k$ th reactor.

\subsection{Formulation of reaction rates}

The ASM \#1 model includes eight fundamental processes. These are: aerobic and anoxic growth of heterotrophic biomass, death of heterotrophic biomass, aerobic growth of autotrophic biomass, decay of autotrophic biomass, ammonification of soluble organic nitrogen, and hydrolysis of both entrapped particulate organic matter and entrapped organic nitrogen. These processes describe nitrogen and chemical oxygen demand within suspended-growth treatment processes, including mechanisms for nitrification and denitrification

The rate functions for these processes are specified in a column vector. The $i$ th row of this vector is written $\mathbf{f}_{i}\left(\mathbf{S}_{k}, \mathbf{X}_{k}\right)$. Thus the $i$ th reaction rate in reactor $k$ depends upon the concentration of the soluble $\left(\mathbf{S}_{k}\right)$ and particulate components $\left(\mathbf{X}_{k}\right)$ in the reactor. In this formulation the variable $\mathbf{S}_{k}$ includes all six of the soluble components. The reaction rate expression may contain as few as one of the twelve state variables. Furthermore, two of the state variables appear in no reaction rate expressions.

For each of the twelve state variables there is a stoichiometric column vector $\alpha_{i, j}$. The second index $(j=$ $1,2, \ldots 12)$ identifies which of the twelve state variables is being considered. The first index $(i=1,2, \ldots)$ indicates which of the eight fundamental processes is being considered. If the entry $\alpha_{i, j}$ is positive, then component $j$ is a product in reaction $i$. If the entry $\alpha_{i, j}$ is zero, then component $j$ is neither a product nor a reactant in reaction $i$. If the entry $\alpha_{i, j}$ is negative, then component $j$ is a reactant in reaction $i$.

\subsection{Characterisation of the activated sludge process}

The concentrations of each of the twelve state variables is determined in each of four reactors. Thus there are potentially forty-eight state variables of interest. In addition, various linear combinations of the state variables 
are of particular interest. These process variables are the chemical oxygen demand (COD), the total suspended solids (TSS), total nitrogen (TN) and total inorganic nitrogen. These process variable may be evaluated in the influent stream, in each of the four reactors, in the wastage stream, and in the effluent stream.

In this paper we only consider the total nitrogen concentration in the effluent stream.

\section{MODEL EQUATIONS}

\subsection{Model equations in the first reactor}

Rate of change of soluble components (excluding oxygen) in the first reactor $(j=1 \ldots 5)$

$$
\frac{\mathrm{d} S_{j, 1}}{\mathrm{~d} t}=\frac{1}{\tau_{1}}\left(S_{j, \text { in }}-S_{j, 1}\right)+\frac{R}{\tau_{1}}\left(S_{j, 4}-S_{j, 1}\right)+\frac{R_{41}}{\tau_{1}}\left(S_{j, 4}-S_{j, 1}\right)+\sum_{i=1}^{8} \alpha_{i, j} \cdot \mathbf{f}_{i}\left(\mathbf{S}_{1}, \mathbf{X}_{1}\right) .
$$

Rate of change of soluble oxygen in the first reactor

$$
\begin{aligned}
\frac{\mathrm{d} S_{\mathrm{O}, 1}}{\mathrm{~d} t}= & \frac{1}{\tau_{1}}\left(S_{O, \text { in }}-S_{\mathrm{O}, 1}\right)+\frac{R}{\tau_{1}}\left(S_{\mathrm{O}, 4}-S_{\mathrm{O}, 1}\right)+\frac{R_{41}}{\tau_{1}}\left(S_{\mathrm{O}, 4}-S_{\mathrm{O}, 1}\right)+K_{\mathrm{L}, \mathrm{a}, 1}\left(S_{\mathrm{O}, \max }-S_{\mathrm{O}, 1}\right) \\
& +\sum_{i=1}^{8} \alpha_{i, O} \cdot \mathbf{f}_{i}\left(\mathbf{S}_{1}, \mathbf{X}_{1}\right) .
\end{aligned}
$$

Rate of change of particulate components in the first reactor $(l=1 \ldots 6)$

$$
\frac{\mathrm{d} X_{l, 1}}{\mathrm{~d} t}=\frac{1}{\tau_{1}}\left(X_{l, \text { in }}-X_{l, 1}\right)+\frac{R}{\tau_{1}}\left(\mathcal{C} X_{l, 4}-X_{l, 1}\right)+\frac{R_{41}}{\tau_{1}}\left(X_{l, 4}-X_{l, 1}\right)+\sum_{i=1}^{8} \alpha_{i, l} \cdot \mathbf{f}_{i}\left(\mathbf{S}_{1}, \mathbf{X}_{1}\right) .
$$

In these equations the parameters are: $\mathcal{C}$, the concentration factor for particulates in the settling unit; $K_{\mathrm{L}, \mathrm{a}, 1}$, the oxygen transfer coefficient in the first reactor; $R$, the recycle ratio of the settling unit; $R_{41}$, the recycle ratio of the recycle unit; $S_{j \text {,in }}$, the concentration of soluble component $j$ in the feed; $S_{O \text {,in }}$, the concentration of soluble oxygen in the feed; $S_{0, \max }$, the maximum concentration of soluble oxygen; $X_{l \text {,in }}$ is the concentration of the particulate components $l$ in the feed, and $\tau_{1}$, the hydraulic retention time in the first reactor.

\subsection{Model equations in reactors two to four}

Rate of change of soluble components (excluding oxygen) in the $k$ th reactor $(j=1 \ldots 5)$

$$
\frac{\mathrm{d} S_{j, k}}{\mathrm{~d} t}=\frac{1+R+R_{41}}{\tau_{k}}\left(S_{j, k-1}-S_{j, k}\right)+\sum_{i=1}^{8} \alpha_{i, j} \cdot \mathbf{f}_{i}\left(\mathbf{S}_{k}, \mathbf{X}_{k}\right)
$$

Rate of change of soluble oxygen in the $k$ th reactor

$$
\begin{aligned}
\frac{\mathrm{d} S_{\mathrm{O}, k}}{\mathrm{~d} t}= & \frac{1+R+R_{41}}{\tau_{1}}\left(S_{O, k-1}-S_{\mathrm{O}, k}\right)+K_{\mathrm{L}, \mathrm{a}, k}\left(S_{\mathrm{O}, \max }-S_{\mathrm{O}, k}\right) \\
& +\sum_{i=1}^{8} \alpha_{i, O} \cdot \mathbf{f}_{i}\left(\mathbf{S}_{k}, \mathbf{X}_{k}\right) .
\end{aligned}
$$

Rate of change of particulate components in the $k$ th reactor $(l=1 \ldots 6)$

$$
\frac{\mathrm{d} X_{l, k}}{\mathrm{~d} t}=\frac{1+R+R_{41}}{\tau_{k}}\left(X_{l, k-1}-X_{l, k}\right)+\sum_{i=1}^{8} \alpha_{i, l} \cdot \mathbf{f}_{i}\left(\mathbf{S}_{k}, \mathbf{X}_{k}\right) .
$$

In these equations the new parameters are: $K_{\mathrm{L}, \mathrm{a}, k}$, the oxygen transfer coefficient in the $k$ th reactor; and $\tau_{k}$, the hydraulic retention time in the $k$ th reactor. 
M.I. Nelson and F.I. Hai, Nitrogen removal in a cascade of four reactors employing the activated sludge process

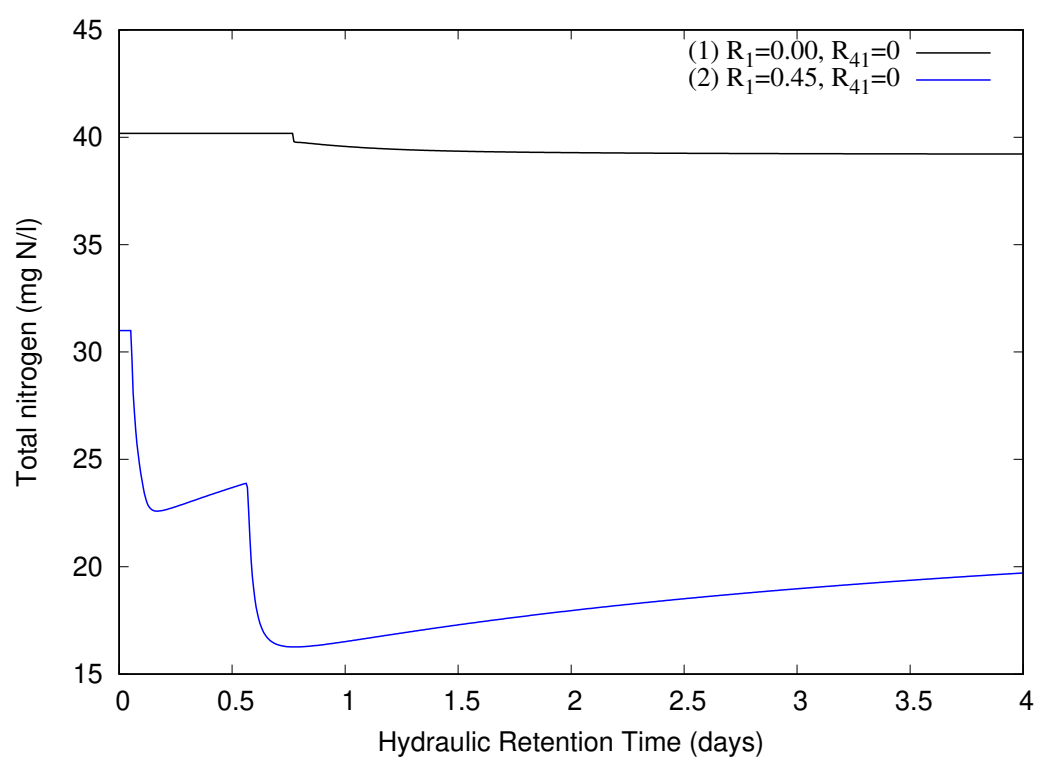

Figure 1. Total nitrogen in the effluent stream leaving the reactor cascade (mg N/l) as a function of the hydraulic retention time (days). The black line represents a reactor cascade without either a settling unit or a recycle unit. The blue line represents a reactor cascade with a settling unit. There is no aeration in the first reactor. The value of the oxygen transfer coefficient in the remaining reactors is $K_{\mathrm{L}, \mathrm{a}, k}=108$ day $^{-1}$.

\section{RESULTS}

\subsection{Assumptions}

We assume that the volumes of each reactor in the cascade are identical. It follows that the hydraulic retention time in each reactor is one-quarter of the total hydraulic retention time through the cascade. The default value of the oxygen transfer coefficients is $K_{\mathrm{L}, \mathrm{a}, k}=108$ day $^{-1}$ (Fikar et al., 2005). We investigate a configuration in which there is no aeration to the first bioreactor. Aeration to the second and bioreactor may be turned off to promote anaerobic conditions.

\subsection{Total nitrogen in the effluent stream: the effect of deploying a settling unit}

Figure 1 shows the total nitrogen in the effluent stream for two reactor configurations. Although neither of these designs is realistic, they provide a useful starting point for our analysis. For each configuration we assume that the first reactor is not aerated whilst the remaining reactors are fully aerated using the default value for the oxygen transfer coefficient.

The black line indicates a process configuration deploying neither a settling unit nor a recycle unit. This is the activated sludge process without the activated sludge. We see that there is insignificant nitrogen removal. Although not applicable within the context of the operation of a wastewater treatment plant, it is provided as a base line from which to assess the improvement in performance that is achieved by adding a settling unit.

The blue line indicates a process configuration deploying only a settling unit. Although this is known not to be an efficient design — which is why the model includes both a settling unit and a recycle unit — it shows that the addition of a settling unit results in a significant decrease in the total nitrogen in the effluent stream. For sufficiently small values of the hydraulic retention time the system evolves onto the washout branch. Along this branch of solutions both forms of biomass are zero. As the hydraulic retention time is increased through the value $\mathrm{HRT} \approx 0.05$ day a transcritical bifurcation occurs following which heterotrophic biomass become viable. A second transcritical bifurcation occurs at HRT $\approx 0.55$ day following which autotrophic biomass becomes viable. (The first transcritical bifurcation is just visible in figure 1 .

The definition of total nitrogen includes nine of the twelve state variables. These can be split into two components: soluble nitrogen, three state variables, and nitrogen contained in particulate matter, nine state variables. 
M.I. Nelson and F.I. Hai, Nitrogen removal in a cascade of four reactors employing the activated sludge process

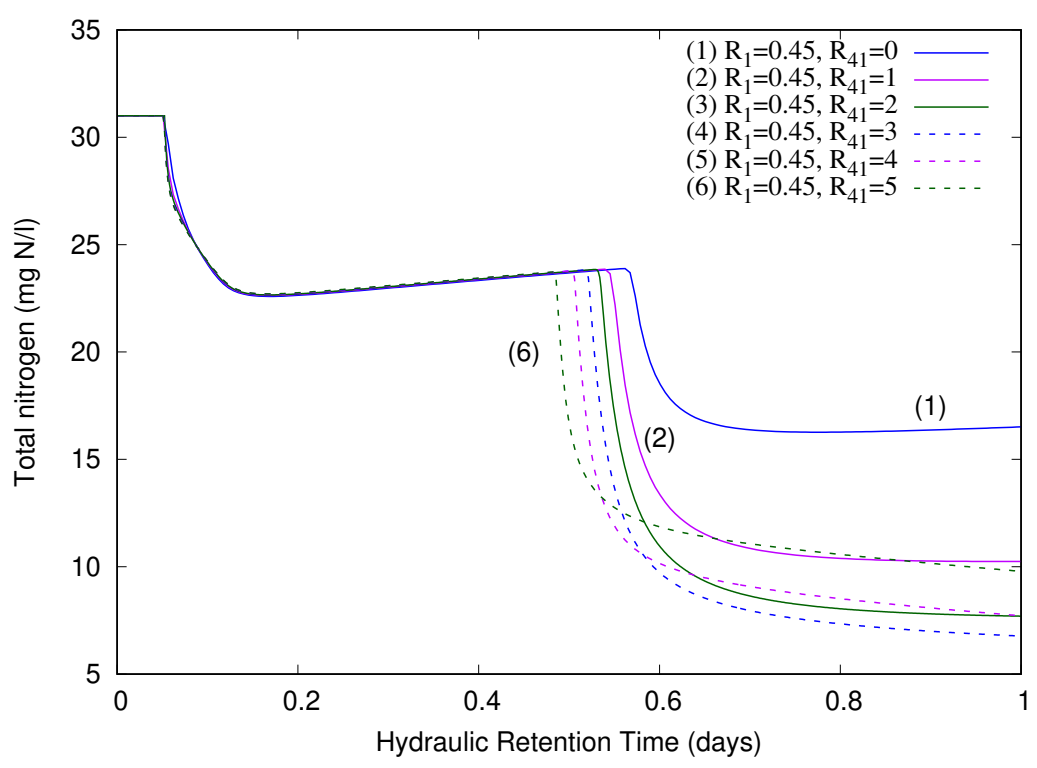

Figure 2. Total nitrogen in the effluent stream leaving the reactor cascade (mg N/l) as a function of the hydraulic retention time (days) in the presence of only a settling unit (line 1) and a settling unit and a recycle unit (lines 2-6). There is no aeration in the first reactor. The value of the oxygen transfer coefficient in the remaining reactors is $K_{\mathrm{L}, \mathrm{a}, k}=108$ day $^{-1}$.

The key assumption underpinning the settling unit model is that it captures all particulate matter. Thus when a settling unit is deployed the effluent concentration only contains soluble nitrogen. This accounts for the fact that the addition of a settling unit lowers the total nitrogen concentration along the washout solution branch.

The range of hydraulic retention times shown on this figure is considerably larger than that used at a wastewater treatment plant. This range has been chosen so as to show that after reaching a minimum value at $\mathrm{HRT} \approx 0.77$ day the total nitrogen concentration in the effluent stream is thereafter an increasing function.

\subsection{Total nitrogen in the effluent stream: the effect of deploying a recycle reactor}

In this section we investigate what happens to the nitrogen concentration in the effluent stream when a recycle reactor is added to a process configuration that includes a settling unit. For each of the configurations we again assume that the first reactor is not aerated whilst the remaining reactors are fully aerated using the default value for the oxygen transfer coefficient.

The solid blue line in figure 2 shows the nitrogen concentration in the effluent stream when there is no recycle unit $\left(R_{41}=0\right)$. This case corresponds to the blue line shown in figure 1 .

The solid purple and green curves correspond to the cases $R_{41}=1$ and $R_{42}=2$ respectively. Comparing the curves for the base case $\left(R_{41}=0\right)$ against these reveals two features of note. Firstly, the value of the hydraulic retention time at which autotrophic biomass become viable decreases as the recycle ratio $\left(R_{41}\right)$ is increased. Secondly, provided that the autotrophic biomass is viable, increasing the value of the recycle ratio decreases the total nitrogen concentration in the effluent stream. Combining these observations we conclude that over the range $0 \leq R_{41} \leq 2$ increasing the recycle ratio of the recycling unit increases the performance of the cascade.

The dashed blue, purple, and green curves correspond to the cases $R_{41}=3, R_{41}=4$, and $R_{41}=5$ respectively. Comparing these curves with the previous curves reveals two features of note. Firstly, the value of the hydraulic retention time at which autotrophic biomass become viable decreases as the recycle ratio $\left(R_{41}\right)$ is increased. Secondly, increasing the value of the recycle ratio does not necessarily improve the performance of the reactor. A consequence of the first observation is that there is always a range of values for which a recycling unit with a high recycle ratio outperforms one with a lower recycle ratio. However, at sufficiently high values of the hydraulic retention time this is no longer true. There is a wide range of retention times over 
M.I. Nelson and F.I. Hai, Nitrogen removal in a cascade of four reactors employing the activated sludge process

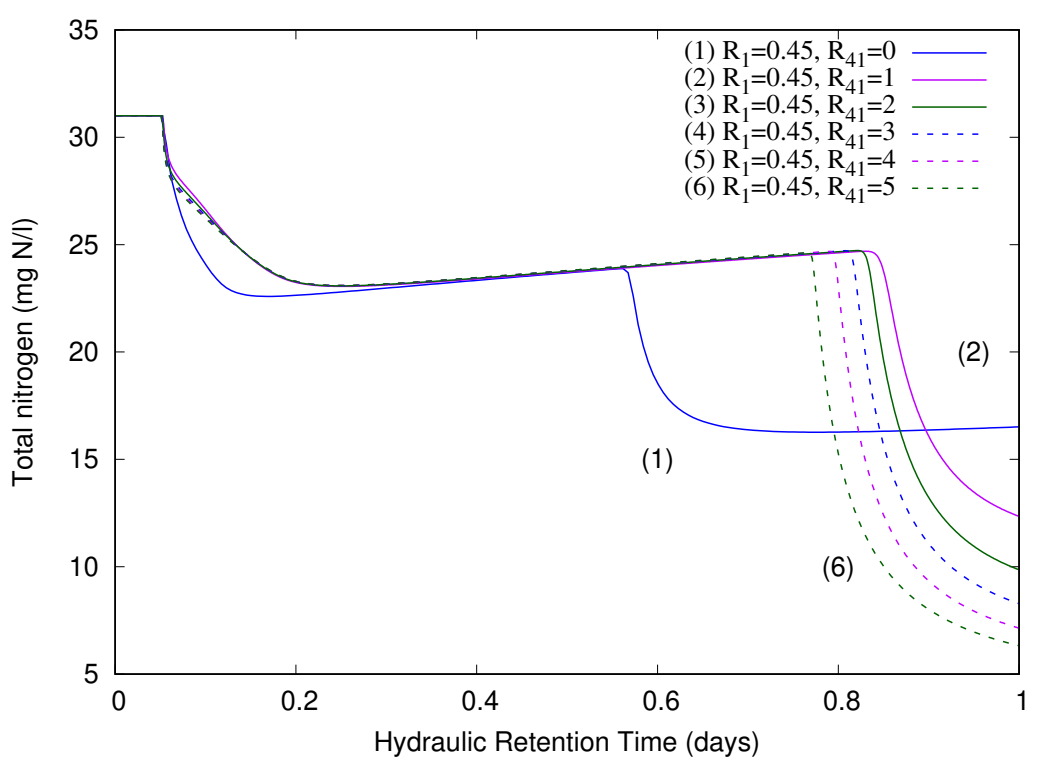

Figure 3. Total nitrogen in the effluent stream leaving the reactor cascade (mg N/l) as a function of the hydraulic retention time (days) in the presence of only a settling unit (line 1) and a settling unit and a recycle unit (lines 2-6). There is no aeration in either the first reactor (all lines) nor the second reactor (except line 1). The value of the oxygen transfer coefficient in the remaining reactors is $K_{\mathrm{L}, \mathrm{a}, k}=108$ day $^{-1}$.

which curve $5\left(R_{41}=4\right)$ is lower than curve $6\left(R_{41}=5\right)$. There is also a wide range of retention times over which curve $4\left(R_{41}=3\right)$ is lower than curve $5\left(R_{41}=4\right)$. The range of hydraulic retention times over which curve $6\left(R_{41}=5\right)$ provides the best performance is quite narrow.

Taken together these results suggest that for a fixed value of the hydraulic retention time the total nitrogen concentration in the effluent stream is minimised at a finite value of the recycle ratio for the recycle unit $\left(R_{41}\right)$ : larger is not necessarily better. It is also clear that the addition of a recycle unit improves the performance relative to a process configuration employing only a settling unit.

\subsection{Total nitrogen in the effluent stream: the effect of deploying a recycle reactor and eliminating aeration in the second reactor}

In this section we investigate what happens to the nitrogen concentration in the effluent stream for the process configurations considered in the previous section when aeration is turned off in the second reactor.

The solid blue line in figure 3 (line 1) shows the nitrogen concentration in the effluent stream when there is no recycle unit $\left(R_{41}=0\right)$ and the second reactor is aerated. This case corresponds to the blue line shown in figures $1 \& 2$.

A strong conclusion from figure 2 is that when the second reactor is aerated the addition of a recycle unit always improves the performance relative to a process configuration employing only a settling unit. This conclusion evidently does not hold when the second reactor is not aerated (lines 2-6) and the comparison is made against a configuration only employing a settling unit in which the second reactor is aerated. In figure 3 it is clear that there is a range of hydraulic retention times for which line 1 provides the lowest nitrogen concentration.

A feature that is common to both figure 2 and figure 3 is that increasing the value of the recycle ratio associated with the recycle unit $\left(R_{41}\right)$ always decreases the value of the hydraulic retention time at which the second transcritical bifurcation occurs. A major point of difference between these two figures is that when the second reactor is not aerated increasing the recycle ratio of the recycle unit always lowers the total nitrogen concentration in the effluent stream. This is not true in figure 2.

From these observations we deduce that when the second reactor is not aerated the recycle ratio of the recycle unit should be increased as high as possible. The question remains as to whether, when a recycle reactor is included in the process configuration, it is better to aerate the second reactor or to turn aeration off? Table 1 
Table 1. Minimum nitrogen concentration ( $\mathrm{mg} \mathrm{N} / \mathrm{l})$ in the effluent stream, for a process employing a settling unit $(R=0.45)$ and a recycle unit as a function of the recycle ratio and of aeration in the second reactor.

$\begin{array}{ccc}R_{41} & \text { Aeration } & \text { No-aeration } \\ 1 & \mathbf{1 0 . 2 4 7} & 12.347 \\ 2 & \mathbf{7 . 6 9 8 5} & 9.8595 \\ 3 & \mathbf{6 . 7 7 2 8} & 8.2819 \\ 4 & 7.7183 & \mathbf{7 . 1 4 3 4} \\ 5 & 9.7841 & \mathbf{6 . 3 2 3 6}\end{array}$

shows the minimum nitrogen concentration achieved in the process configurations represented by lines 2-6 in both figures $2 \& 3$. (These are the minimum values obtained over the interval $0 \leq \mathrm{HRT} \leq 1$. All but one of these values occurs when HRT $=1$ day). From this data we conclude that if the maximum achievable recycle ratio is 'lower' then aeration should be employed in the second reactor. However, if the maximum achievable recycle ratio is 'higher' then aeration should be turned-off in the second reactor.

\section{CONCLUSIONS}

We have investigated a model for the treatment of a wastewater by the activated sludge process. The reactor configuration is a four reactor cascade. The process configurations allows for the placement of a settling unit and a recycle reactor after the fourth reactor of the cascade. The exit streams from these units enter the first reactor of the cascade. (A fraction of the stream leaving the settling unit is wasted and the remainder enters the first reactor). We have primarily investigated the effect of varying the value of the recycle ratio of the recycling unit under two conditions depending upon whether the second reactor is aerated. (It is assumed that the first reactor is not aerated). We have assumed that the size of each reactor in the cascade is equal.

In this paper we have characterised the operation of a wastewater treatment plant by the nitrogen concentration in the effluent stream. As our point of reference we have considered the performance of a process configuration containing a settling unit but no recycle unit in which the second reactor is aerated.

When the second reactor is aerated we have shown that the value of the recycle ratio that minimise the nitrogen concentration depends upon the value of the hydraulic retention time. The overall picture is evidently quite complicated. However, there is a significant range of values for the hydraulic retention time over which the optimal performance is achieved by employing a 'moderate' recycle ratio.

When the second reactor is not aerated we have shown that increasing the value of the recycle ratio always improves performance. Thus the recycle unit should be operated at the maximum attainable value of the recycle ratio. If this value is 'low' then aeration should be maintained to the second reactor. If this value is 'high' then aeration should be turned off.

We are undertaking a more comprehensive analysis in which we are investigating what improvements can be made by varying the volumes of the reactors in the cascade.

\section{REFERENCES}

Fikar, M., B. Chachuat, and M. Latifi (2005). Optimal operation of alternating activated sludge processes. Control Engineering Practice 13, 853-861.

Hauduc, H., L. Rieger, A. Oehmen, M. van Loosdrecht, Y. Comeau, A. Héduit, P. Vanrolleghem, and S. Gillot (2013). Critical review of activated sludge modeling: State of process knowledge, modeling concepts, and limitations. Biotechnology and Bioengineering 110(1), 24-46.

Henze, M., C. Leslie Grady, W. Gujer, G. Maris, and T. Matsuo (1987). Activated sludge process model no 1. Scientific and Technical Report 1, IAWQ, London, UK.

Makinia, J. (2010). Mathematical Modelling and Computer Simulation of Activated Sludge Systems (First ed.). London: IWA Publishing.

Nelson, M., R. Alqahtani, and F. Hai (2018). Mathematical modelling of the removal of organic micropollutants in the activated sludge process: A linear biodegradation model. The ANZIAM Journal 60(2), 191-229.

Nelson, M., H. Sidhu, S. Watt, and F. Hai (2019). Performance analysis of the activated sludge model (number 1). Food and Bioproducts Processing 116, 41-53. 